\title{
1 Alzheimer's disease alters astrocytic functions related to neuronal support and transcellular internalization
}

\section{2 of mitochondria}

3 Riikka Lampinen ${ }^{1}$, Irina Belaya ${ }^{1}$, Liudmila Saveleva ${ }^{1}$, Jeffrey R Liddell ${ }^{2}$, Dzhessi Rait ${ }^{1}$, Mikko T Huuskonen ${ }^{1}$,

4 Raisa Giniatullina ${ }^{1}$, Annika Sorvari ${ }^{1}$, Liisi Soppela ${ }^{1}$, Nikita Mikhailov ${ }^{1}$, Isabella Boccuni ${ }^{1}$, Rashid Giniatullin ${ }^{1}$, 5 Marcela Cruz-Haces ${ }^{1}$, Julia Konovalova ${ }^{3}$, Marja Koskuvi ${ }^{1}$, Tuomas Rauramaa ${ }^{4,5}$, Andrii Domanskyi ${ }^{3}$, Riikka H 6 Hämäläinen ${ }^{1}$, Gundars Goldsteins ${ }^{1}$, Jari Koistinaho ${ }^{1,6}$, Tarja Malm ${ }^{1}$, Sweelin Chew $^{1}$, Kirsi Rilla ${ }^{7}$, Anthony R 7 White $^{8}$, Nicholas Marsh-Armstrong ${ }^{9}$, Katja M Kanninen ${ }^{1}$.

3. Institute of Biotechnology, University of Helsinki, Finland

4. Department of Pathology, Kuopio University Hospital, University of Eastern Finland, Kuopio, Finland.

5. Hemorrhagic Brain Pathology Research Group, NeuroCenter, Kuopio University Hospital, Kuopio, Finland.

6. Neuroscience Center, University of Helsinki, Finland

7. Institute of Biomedicine, University of Eastern Finland, Finland

8. Mental Health Program, Department of Cell and Molecular Biology, QIMR Berghofer Medical Research Institute, Australia

9. Department of Ophthalmology and Vision Science, University of California Davis, USA

\section{Corresponding author}

Katja Kanninen

Associate professor, Neurobiology of Disease research group leader

A.I. Virtanen Institute for Molecular Sciences, University of Eastern Finland

Neulaniementie 2, 70210 Kuopio, Finland 


\section{Abstract}

Under physiological conditions in vivo astrocytes internalize and degrade neuronal mitochondria in a process called transmitophagy. Mitophagy is widely reported to be impaired in neurodegeneration but it is unknown whether and how transmitophagy is altered in Alzheimer's disease (AD). Here we report that the internalization and degradation of neuronal mitochondria are significantly increased in astrocytes isolated from aged AD mouse brains. We also demonstrate for the first time a similar phenomenon between human neurons and AD astrocytes, and in murine hippocampi in vivo. The results suggest the involvement of S100a4 in impaired mitochondrial transfer between neurons and aged AD astrocytes. Significant increases in the mitophagy regulator Ambra1 were observed in the aged $\mathrm{AD}$ astrocytes. These findings demonstrate altered neuron-supporting functions of aged AD astrocytes and provide a starting point for studying the molecular mechanisms of transmitophagy in AD.

\section{Keywords}

Alzheimer's disease; astrocytes; mitochondria; mitophagy; transmitophagy

\section{Introduction}

Alzheimer's Disease (AD) is a major cause of dementia, a progressive neurodegenerative disorder with increased risk upon aging. The pathology of the AD is typified by extracellular beta-amyloid plaques (A $\beta$ ), intracellular neurofibrillary tangles, neuronal loss, neuroinflammation and oxidative stress. However, despite extensive research, the actual causes of neurodegeneration remain unclear and there is no cure for the disease. (Lane et al., 2018)

The brain is an organ with an exceptionally high energy need. The mitochondria are central for energy metabolism, converting glucose to adenosine triphosphate (ATP) via oxidative phosphorylation. Glucose is considered to be the main energy source for neurons, and thus the brain is highly sensitive to changes in the mitochondrial function of cells. In addition, mitochondria are central for maintaining calcium homeostasis and various cell signaling pathways. Given that upon damage mitochondria release apoptotic factors such as cytochrome c, and that the mitochondria are a major source of reactive oxygen species (ROS), maintenance of healthy mitochondria is highly 
important for cellular well-being. Mitochondria quality is controlled by fusion and fission, intracellular localization, and permanent degradation of dysfunctional mitochondria via mitophagy. Impairments in mitophagy, selective form of autophagy, cause the intracellular accumulation of damaged mitochondria and are associated with adverse effects for the health of the brain cells. Cells with especially high risk are the long-lived neuronal cells. (Wang et al., 2020)

Mitochondrial dysfunction is associated with AD-induced neurodegeneration and aging. Synaptic mitochondria accumulate beta-amyloid $(\mathrm{A} \beta)_{1-40}$ and $\mathrm{A} \beta_{1-42}$, leading to impaired mitochondrial function and dynamics in neurons (Wang et al., 2016) . A reduction in mitochondrial number and altered phenotypes are observed in the presynaptic regions of neurons of $\mathrm{AD}$ patients (Pickett et al., 2018). Given that neurons are post-mitotic cells, they also accumulate dysfunctional mitochondria upon aging. Furthermore, defective mitophagy is observed in AD-affected neurons (Ye et al., 2015) and AD patient brains have reported to contain reduced levels of autophagy-inducing protein beclin 1 (Pickford et al., 2008). Deficiency in beclin 1 have been shown to enhance A $\beta$ deposition in mice modeling AD as well (Pickford et al., 2008).

The majority of studies deciphering aging- and AD-associated changes in mitochondria have in the past focused on neurons. Only relatively recently, astrocytes have started to gain larger interest due to their essential roles in maintaining brain health and implications in disease. Astrocyte reactivity is a common feature observed both in $\mathrm{AD}$ and upon aging (Clarke et al., 2018; Liddelow et al., 2017). Accumulation of $\mathrm{A} \beta_{1-42}$ causes adverse effects in astrocytes by impairing their mitochondrial function (Yao et al., 2018) and causing autophagy inhibition (Hong et al., 2018). However, to date, there are no reports assessing mitophagy in AD-affected astrocytes.

To meet the requirements for functional mitochondria, many cell types have been reported to be capable of transcellular mitochondrial movement. For example, transfer of mitochondria from mesenchymal stem cells to damaged or stressed cells of various cell types has been shown to be an important means of cellular regeneration and repair (Soundara Rajan et al., 2020) . Tunneling nanotubes (TNTs) are suggested as one possible means of mitochondrial transfer between cells (Rustom et al., 2004; Soundara Rajan et al., 2020). Transmitophagy, the transfer of mitochondria to neighboring cells specifically for degradation, was termed for the first time by (Davis 
et al., 2014). The authors reported that degradation of retinal ganglion cell axon mitochondria occurs inside adjacent astrocytes under normal physiological conditions. Recently, mitophagy of dopaminergic neuron mitochondria was also suggested to be completed in neighboring astrocytes via spheroid-mediated transmitophagy. It was suggested that the aging of the astrocytes could lead to a failure in spheroid-mediated transmitophagy and in this way take part in the pathogenesis of Parkinson's disease. (Morales et al., 2020)

Astrocytes are acknowledged to be critical for brain health, and to be affected by neurodegenerative diseases, but their functions related to mitochondria are less studied. The field is lacking detailed knowledge on the role of astrocytic mitophagy and transmitophagy in $\mathrm{AD}$. Here we assessed how $\mathrm{AD}$ alters astrocytic functions related to neuronal support with a specific focus on mitochondria-related mechanisms, including transmitophagy. We were interested whether the AD-affected astrocytes are capable of internalizing and degrading neuronal mitochondria and whether the process is possibly altered due to AD-related changes in the basic functions of the astrocytes or their mitochondria.

\section{Material and methods}

\section{1. Animals}

All experiments were approved by the National Animal Experiment Board of Finland and performed according to the animal protection guidelines of the Council of the European Union. The mouse lines used in this study were 5xFAD (on a C57BL/6J background) and C57BL/6J. The 5xFAD mice express human amyloid precursor protein (APP) and presenilin 1 (PS1) as transgenes with a total of five mutations causing familial AD (FAD) in these transgenes, three mutations in the APP (K670N/M671L, I716V, and V717I) and two in the PS1 (M146L and L286V) (Oakley et al., 2006).

\subsection{MitoEGFPmCherry and GFP lentiviral constructs}

The plasmid for MitoEGFPmCherry was kindly provided by Professor Marsh-Armstrong, University of California, Davis (Davis et al., 2014). The plasmid was used to re-clone the transgenes to pCDH lentivirus transfer vector, in order to produce third generation lentiviral vectors. The production and purification of the lentiviral 
vectors were carried out at the laboratory of Docent Andrii Domanskyi, University of Helsinki. In the lentiviral vector the transgene expression was driven by the human synapsin (hSYN) gene promoter. The construct used in this study for astrocytes took advantage of a human phosphoglycerate kinase (hPGK) promoter to drive ubiquitous expression of green fluorescent protein (GFP) in the cells. This was produced by the Biocenter Kuopio Viral Gene Transfer Core.

\subsection{Primary adult astrocyte cultures}

The primary astrocytes were harvested from 2-3-, 5-6- and 10-12-month-old (mo) 5xFAD mouse brains as described in (Iram et al., 2016; Konttinen et al., 2019) with the following modifications. Briefly, the brains without cerebellum, olfactory bulb and brainstem were dissociated to single cell suspension with Adult Brain Dissociation kit (Miltenyi Biotech, Bergisch Gladbach, Germany) following the manufacturer's instructions. The cells were cultured in DMEM/F-12 with GlutaMAX supplemented with 10\% iFBS and 1\% P/S (all Thermo Fisher Scientific , Waltham, MA, USA) on poly-D-lysine (Sigma-Aldrich, Saint Louis, MO, USA) coated 6-wells. The medium was changed daily for the 7 first days. The cells were split on day 7-8 in vitro (DIV) and on 10-11 DIV to expand the cultures. The cells were used for experiments at 14-26 DIV. 0.25\% Trypsin-EDTA was used for detaching the cells. For studying internalization of neuronal mitochondria by astrocytes, the primary astrocytes were transduced with the lentivirus vector driving GFP expression in the cells with MOI 5 or 7 for 48 hours. The proportion of different cell types in the murine adult astrocyte cultures were assessed with qPCR and immunostaining for several markers of astrocytes, neurons, and microglia. The adult astrocyte cultures contain more than 80-85\% of astrocytes and a small proportion of other glial cells. Neurons were not detected in the cultures.

\subsection{Primary cortical neuronal cultures}

Primary cortical neuron cultures were prepared using the cortices of C57BL/6J mice on embryonic day 15 as described in (Loppi et al., 2021). The cells plated for experiments on poly-D-lysine coated plates $(10 \mu \mathrm{g} / \mathrm{ml} \mathrm{in}$ water, Sigma-Aldrich) in Neurobasal media supplemented with 2\% B27, $1 \%$ penicillin-streptomycin $(10,000$ U/ml) (all Thermo Fisher Scientific) and $0.5 \mathrm{mM}$ L-glutamine (Lonza, Walkersville, MD, USA). On 4 or 5 DIV, 
122

123

124

125

126

127

128

129

130

131

132

133

134

135

136

137

138

139

140

141

142

143

144

145

half of the media was replaced with fresh media to supply the cells with efficient amount of nutrients, unless the neurons were transduced or stained with mitochondria-targeted dye already on 2 DIV. The cells were used for experiments on 6-7 DIV.

\subsection{Primary neuron-astrocyte co-cultures}

Primary cortical neurons were plated on 24-well plates in Neurobasal media supplemented with 2\% B27, $1 \%$ penicillin-streptomycin (10,000 U/ml) (all Thermo Fisher Scientific) and 0.5 mM L-glutamine (Lonza) with 200000 neurons/well for the MTT assay and 120000 neurons/well for live cell imaging with rhodamine 123 dye. Primary astrocytes were seeded on 24-well transwells with $0.4 \mu \mathrm{m}$ pore size (Sarstedt AG \& Co. Nümbrecht, Germany) with 5000 astrocytes/transwell for 1-2 days before transferring the transwells on top of the wells with cortical neurons. The two cell types were co-cultured for a total of 2 days before experiments were carried out. The cells were exposed to $250 \mu \mathrm{M}$ glutamate (Sigma-Aldrich) for 24 hours before the MTT assay or live cell imaging with rhodamine 123 dye.

For assessing the internalization and degradation of neuronal mitochondria by astrocytes, the primary neurons were plated in $\mu$-Slide 8 wells at 100000 neurons/well (ibidi GmbH, Gräfelfing, Germany). At 2 DIV, the neuronal cells were either transduced with the LV-mito-EGFP-mCherry-msSODUTR with MOI 1 for 24 hours or stained for 30 minutes at $+37^{\circ} \mathrm{C}$ with $100 \mathrm{nM}$ MitoTracker Red CMXROS dye (Thermo Fisher Scientific). Three days after labeling the neuronal mitochondria either with the lentivector or MitoTracker, the primary astrocytes were seeded in co-cultures with the neurons. The cells were grown in co-cultures for 48 hours before fixing the cells with $4 \%$ formaldehyde.

\section{6. iPSC-derived neuron- and astrocyte-like cultures}

The induced pluripotent stem cells (iPSCs) used in this study were derived from control subjects described previously (Tiihonen et al., 2019). The iPSC lines were generated and differentiated to astroglial cells and neuronal cells using previously described protocols (Oksanen et al., 2017; Tiihonen et al., 2019). On day 4, after plating the neuronal cells as 75000 cells $/ \mathrm{cm}^{2}$ onto poly-ornithinine/Matrigel-coated glass coverslips, the neuronal cells were 
transduced with the LV-mito-EGFP-mCherry-msSODUTR at MOI 1 for 24 hours. The astrocyte progenitor cells derived from spheres were further maturated for one week in astrodifferentiation medium (DMEM/F12 supplemented with $1 \% \mathrm{~N}_{2}$ supplement, $1 \%$ Glutamax, $1 \%$ non-essential amino acids, $0.5 \%$ penicillin/streptomycin (50 IU/50 $\mu \mathrm{g} / \mathrm{mL}$ ), $0.5 \mathrm{IU} / \mathrm{mL}$ heparin (LEO Pharma, Ballerup, Denmark), $10 \mathrm{ng} / \mathrm{mL} \mathrm{bFGF}$ and $10 \mathrm{ng} / \mathrm{mL}$ EGF (both growth factors from PeproTech EC Ltd., London, UK) prior to plating in co-culture with neurons. Following maturation, the astrocytes were detached with Accutase (STEMCELL Technologies, Vancouver, Canada) and replated in neural sphere medium at 10000 astrocytes $/ \mathrm{cm}^{2}$ on top of the lentivector-transduced neurons. Cells were grown as mixed cultures for 7 days prior to fixing the cultures with $4 \%$ paraformaldehyde.

\subsection{Intracerebral injections of MitoEGFPmCherry lentivirus}

In vivo, the localization of neuronal mitochondria inside 5xFAD mouse astrocytes was assessed following intracerebral injection of the LV-mito-EGFP-mCherry-msSODUTR to the hippocampi of 6 mo 5xFAD mice. The vector was injected in the volume of $2.5 \mu \mathrm{l}$ into the dentate gyrus region of the hippocampus by using the following coordinates: $\pm 3.2 \mathrm{~mm}$ medial/lateral, $-2.7 \mathrm{~mm}$ anterior/posterior, $-2.7 \mathrm{~mm}$ dorsal/ventral from the bregma as described previously (Kanninen et al., 2009). One week after the intracerebral injection with the viral vector, the mice were deeply anesthetized, perfused transcardially with heparinized saline and the brains were immersionfixed in $4 \%$ PFA for $22 \mathrm{~h}$ as described previously (Kanninen et al., 2009). Following cryoprotection in sucrose solution, the brain tissues were frozen in liquid nitrogen and cut as $20 \mu \mathrm{m}$ sections with cryostat (Leica Microsystems GmH, Wetzlar, Germany).

\subsection{Immunocytochemistry}

The co-cultures of lentivector-transduced murine primary neurons and astrocytes were fixed with $4 \%$ formaldehyde in DPBS for 20 minutes, permeabilized with $0.2 \%$ Triton X-100 in DPBS for 30 minutes and the nonspecific binding of antibodies was blocked with incubation with 5\% normal goat serum in DPBS for 30 minutes at room temperature. For immunostaining of the astrocytes, the co-cultures were incubated first with primary antibody for glial fibrillary acidic protein (GFAP, 1:400, Z033429-2, Dako, Glostrup, Denmark) prepared in 5\% 
NGS in DPBS overnight at $+4^{\circ} \mathrm{C}$ following an incubation with Alexa Fluor405 (A31556, Thermo Fisher Scientific, 1:500) or Alexa Fluor680 (A31556, Thermo Fisher Scientific, 1:2000) secondary antibodies prepared in 5\% NGS in DPBS for $2 \mathrm{~h}$ at room temperature. For iPSC-derived neuron- and astrocyte-like cultures, the protocol for immunocytochemistry was similar with only minor modifications. The co-cultures were fixed with $4 \%$ PFA, permeabilized with $0.25 \%$ Triton X-100 in DPBS for $1 \mathrm{~h}$ at room temperature and the blocking with $5 \%$ normal goat serum was extended for 1h. The primary antibody against GFAP (Dako, Z033429-2) was used at 1:500 dilution and Alexa Fluor405 secondary antibody at 1:500 dilution. The coverslips were mounted on glass slides with Vectashield mounting medium (Vector Laboratories INC, Burlingame, CA, USA) for fluorescence with 4',6diamidino-2-phenylindole (DAPI).

For visualizing tunneling nanotubes, the co-cultures of MitoTracker CMXROS labeled primary E15 neurons and neurons with Mitotracker CMXROS dye.

\subsection{Immunohistochemistry}

Mouse brain cryosections for studying transmitophagy in vivo were blocked with $10 \%$ normal goat serum for 30 
To study neuronal localization of S100a4 in the hippocampal area of 12 mo WT and 5xFAD mice, the mice were deeply anesthetized and transcardially perfused as described above. The brains were post-fixed in $4 \%$ PFA for $21 \mathrm{~h}$ at $+4{ }^{\circ} \mathrm{C}$, cryoprotected with immersion to $30 \%$ sucrose for $48 \mathrm{~h}$ at $+4{ }^{\circ} \mathrm{C}$ and finally frozen at $-70{ }^{\circ} \mathrm{C}$ prior sectioning. The $20 \mu \mathrm{m}$ sagittal sections of one hemisphere were cut with $400 \mu \mathrm{m}$ interval. The sections were rehydrated overnight in 0.1 M PB and washed with 1xPBS before antigen retrieval with boiling the sections in $10 \mathrm{mM}$ citrate. Endogenous peroxidases were blocked by treating sections with $0.3 \% \mathrm{H}_{2} \mathrm{O}_{2}$ in methanol for 30 minutes. Next, the sections were blocked with 0.5\% Mouse on Mouse Blocking Reagent (Vector Laboratories, MKB-2213) for 1h at

RT with following blocking with TSA blocking reagent (Perkin Elmer, Waltham, MA, USA, FP1020) as 0.5\% solution in PBS pH 7.4 for another 1h at RT. Sections were incubated o/n at RT with primary antibodies diluted in $0.5 \%$ solution of the TSA blocking reagent. Primary antibodies used for assessing the neuronal localization of S100a4 were anti-NeuN (MAB377, Sigma-Aldrich, dilution 1:200) and anti-S100a4 (ab41532, Abcam, Cambridge, UK, dilution 1:100). Incubation with the secondary antibodies was performed for $2 \mathrm{~h}$ at $\mathrm{RT}$. Secondary antibodies biotin conjugated goat anti-rabbit (BA-1000, Vector Laboratories, dilution 1:200) and goat anti-mouse IgG $(\mathrm{H}+\mathrm{L})$ Alexa Fluor 488 (A11001, Thermo Fisher Scientific, dilution 1:250) were diluted in 0.5\% solution of the TSA blocking reagent. Sections were further processed according to the instructions in the TSA Plus Cyanine 3 kit (Perkin Elmer, NEL744001KT) in order to visualize the biotin conjugated secondary antibody bound to antiS100a4 primary antibody. Lastly, the sections were embedded with Vectashield mounting medium (Vector Laboratories) for fluorescence with 4',6-diamidino-2-phenylindole (DAPI). For visualizing S100a4 together with astrocytic marker GFAP and amyloid plaques, same protocol was used with anti-S100a4 (ab41532, Abcam, dilution 1:100), anti-GFAP (ab4674, Abcam, dilution 1:2500) and Anti-Amyloid $\beta$, clone W0-2 (MABN10, Sigma-Aldrich, dilution 1:1000) primary antibodies. Secondary antibodies used in this staining were biotin conjugated goat anti-rabbit (BA-1000, Vector Laboratories, dilution 1:200), goat anti-chicken IgY (H+L) Alexa 
218 To study neuronal localization of S100a4 in the human brain, paraffin blocks from posterior hippocampus were cut to $5 \mu \mathrm{m}$ sections and anti-S100a4 immunostaining was combined with Nissl-staining. There were two samples from patients with Alzheimer's disease and two control cases in the staining. The study was approved by the Ethics Committee of the Hospital District of Northern Savonia (276/2016). First, the paraffin sections were deparaffinized using xylene and rehydrated with decreasing concentrations of ethanol. Next, the sections were washed with 1xPBS before antigen retrieval with boiling the sections in $10 \mathrm{mM}$ citrate. Endogenous peroxidases were blocked by treating sections with $0.3 \% \mathrm{H}_{2} \mathrm{O}_{2}$ in methanol for 30 minutes and followed with blocking with TSA blocking reagent (Perkin Elmer) as $0.5 \%$ solution in PBS pH 7.4 for $1 \mathrm{~h}$ at RT. Sections were incubated o/n at RT with primary antibodies diluted in $0.5 \%$ solution of the TSA blocking reagent. The primary antibody used was antiS100a4 (ab41532, Abcam, dilution 1:100). Incubation with the secondary antibody (Biotin conjugated goat antirabbit (BA-1000, Vector Laboratories, dilution 1:200)) diluted in $0.5 \%$ solution of the TSA blocking reagent was performed for $2 \mathrm{~h}$ at RT. Sections were further processed according to the instructions in the TSA Plus Cyanine 3 kit (Perkin Elmer, NEL744001KT) to visualize the biotin conjugated secondary antibody bound to anti-S100a4 primary antibody. The sections were imaged for representative images without letting the sections to dry with Zeiss Axio Imager 2 fluorescent microscope with 10x objective before continuing the Nissl-staining. The Nisslstaining was performed by first rinsing the sections with water, followed with incubation with solution of thionin acetate, after which the sections were washed twice with water and once with both $50 \%$ and $70 \%$ ethanol. Finally, the sections were mounted with xylene before imaging for Nissl for the same locations as the images for antiS100a4 staining were taken previously.

Stained sections were imaged with Zeiss Axio Imager 2 fluorescent microscope with 10x objective for all the immunohistochemical staining's of S100a4 and ZEN software (Carl Zeiss AG). Images were analyzed with ImageJ for quantification of percentage of immunoreactive area for S100a4 in selected regions. 
242 Transmitophagy and internalization of the lentivirus labelled neuronal mitochondria in vitro and in vivo were

243 visualized by imaging with a Zeiss Axio Observer inverted microscope with LSM800 confocal module with 63x 244 objective and ZEN software v. 2.3 (Carl Zeiss AG, Oberkochen, Germany). For quantifying the internalized and 245 degraded neuronal mitochondria in primary murine or iPSC-derived astrocytes, the number of intact and degraded neuronal mitochondria were manually counted per one astrocyte from confocal z-stack images aided with the 247 profile tool in the ZEN software. The results were counted as an average of three biologically individual experiments for each age-group for murine astrocytes and as per one donor for iPSC-astrocytes.

\subsection{Measurement of neuronal metabolic activity}

Metabolic activity of the neurons co-cultured with astrocytes isolated from 5-6 mo and 11-12 mo WT or 5xFAD measured at $595 \mathrm{~nm}$ with Wallac Victor 1420 microplate reader (Perkin Elmer).

\subsection{Measuring mitochondrial membrane potential with rhodamine 123 imaging}

Cell cultures (astrocytes or neurons) were incubated for $30 \mathrm{~min}$ at $37^{\circ} \mathrm{C}$ in $5 \mu \mathrm{M}$ rhodamine 123 solution (ThermoFisher Scientific, $5 \mathrm{mM}$ stock solution in $99 \% \mathrm{EtOH}$ ). Then cells were transferred to the imaging system glucose, $2.5 \mathrm{mM} \mathrm{KCl}, 2 \mathrm{CaCl}_{2}, 1 \mathrm{MgCl}_{2}$. $\mathrm{pH}$ was adjusted to 7.4). First, the baseline was recorded for $1 \mathrm{~min}$ before applying $4 \mu \mathrm{M}$ FCCP (Abcam, $20 \mathrm{mM}$ stock solution in DMSO) for $2 \mathrm{~min}$. The response was calculated as $\Delta \mathrm{F} / \mathrm{F}_{0}$ (normalized to baseline). Both BSS and FCCP solution contained $0.02 \%(\mathrm{v} / \mathrm{v})$ DMSO. Our TILL Photonics imaging system (TILL Photonics GmbH, Germany) was equipped with fast perfusion system (Rapid Solution 
applying solutions ( $30 \mathrm{~ms}$ ). Cells were imaged with Olympus IX-70 (Olympus Corporation, Tokyo, Japan) with

CCD camera (SensiCam, PCO imaging, Kehlheim, Germany) with 10x objective for astrocytes or 20x objective

for neurons. The excitation wavelength was $495 \mathrm{~nm}$. Imaging was conducted at 1 FPS. All the experiments were conducted using Live Acquisition and processed with Offline Analysis software (TILL Photonics GmbH, Munich,

Germany).

\subsection{Western blotting}

272

Cells were lysed directly in 1x Laemmli buffer (62.5 mM Tris- $\mathrm{HCl}$ (pH 6.8), 2.3\% SDS, 5\% $\beta$-mercaptoethanol,

For detection of the proteins of interest, the membranes were incubated for $2 \mathrm{~h}$ at room temperature in HRP conjugated IgG anti-rabbit secondary antibody (Bio-Rad 170-65-15, 1:3000) or Cy5 conjugated IgG anti-mouse secondary antibody (Jackson ImmunoResearch Laboratories Europe Ltd., Cambridgeshire, UK 715-175-151, 1:1000 dilution). The membranes incubated with HRP conjugated secondary antibody were further developed using enhanced chemiluminescence (SuperSignal ${ }^{\mathrm{TM}}$ West Pico PLUS Chemiluminescent Substrate, Thermo Fisher Scientific). All membranes were imaged on a Bio-Rad ChemiDoc XRS+ System.

\subsection{Statistical analyses and graphical illustrations}

The data was analyzed using t-test or ANOVA as appropriate using GraphPad Prism 8.1.0 (GraphPad Software Inc, San Diego, CA, USA). Before performing the statistical test, the data was analyzed for normality and possible outliers were identified with the ROUT method $(\mathrm{Q}=1 \%)$ in GraphPad Prism. Statistical significance was assumed 
if $\mathrm{P}<0.05$ and confidence intervals were reported with SEM. The graphical illustrations were created with

BioRender.com.

Material and methods for supplementary figures and tables are described in separate supplementary file.

293

294

295

296

297

298

299

300

301

302

303

304

305

306

307

308

309

310

311

312

313

\section{Results}

\subsection{Transcellular internalization and degradation of neuronal mitochondria is altered in aged AD astrocytes}

Movement of mitochondria between neurons and astrocytes has been shown to occur in normal physiological conditions in the mouse optic nerve head in vivo (Davis et al., 2014), after stroke from astrocytes to neurons both in vitro and in vivo (Hayakawa et al., 2016) and to rescue cisplatin-treated neurons (English et al., 2020) . Here we utilized two fluorescent mitochondrial labels to study whether astrocytes internalize mitochondria derived from neurons, and whether the process is altered in AD. Neuronal mitochondria were labelled with a MitoTracker CMXROS dye, or a mitochondria-targeted tandem fluorophore reporter before co-culturing neurons with astrocytes. Confocal imaging revealed the presence of neuron-derived mitochondria inside both WT and AD astrocytes (Fig 1A and 1B). Astrocytes harvested from 5xFAD mouse brains internalized significantly more neuronal mitochondria than their WT controls in all age groups. The aged (11 mo) 5xFAD astrocytes internalized the most neuronal mitochondria (mean difference $27.13 \pm 6.805, \mathrm{p}=0.0002$ ) compared to the wild-type (WT) astrocytes (Fig 1C). The increased internalization of the neuronal mitochondria by AD astrocytes was also confirmed with human-derived cells. The iPSC-astrocytes derived from a symptomatic AD patient with the PSEN1 $\Delta \mathrm{E} 9$ mutation were observed to internalize significantly more neuronal mitochondria derived from iPSC-neurons compared to its isogenic, PSEN1 mutation corrected, control cells (mean difference 4,960 $\pm 1,554, \mathrm{p}=0.0023$ ). Interestingly, there was no significant difference in internalization of the neuronal mitochondria between the iPSCastrocytes derived from pre-symptomatic AD patient and its isogenic control cells (Fig 1E). Thin nanotube-like structures consisting of filamentous actin were observed to be bridging between the primary neurons and astrocytes in the cultures (Fig. 1F), suggesting one possible route allowing the transcellular movement of mitochondria from 
neurons to astrocytes. The $\mathrm{H}_{2} \mathrm{O}_{2}$ treatment on neurons seemed to increase the thin TNT-like protrusions around astrocytes, and even more in 5xFAD astrocytes compared to WT astrocytes (Fig 1G).

316 To validate whether astrocytes can degrade neuron-derived mitochondria in vitro, we transduced primary neurons

317 with a tandem fluorophore reporter of acidified mitochondria (Fig 2A) before co-culturing the neurons with 318 astrocytes. Confocal imaging revealed the presence of neuron-derived, degraded mitochondria inside the cultured 319 astrocytes (Fig 1B). Transmitophagy was also confirmed in human induced pluripotent stem cell (iPSC) derived astrocytes co-cultured with iPSC-derived neurons (Fig 1D) and in the mouse hippocampi in vivo (Fig 2D). and Oliver, 2019) and AD-affected neurons have previously been reported to accumulate dysfunctional mitochondria (Fang et al., 2019). We next questioned whether transmitophagy alterations occur in AD. Co-cultures of WT neurons with astrocytes harvested from mouse brains at various ages demonstrated that the degree of transmitophagy was not changed in young (2-3 mo) astrocytes. However, AD astrocytes derived from both 6 mo and 11 mo mice displayed an increase (in the total amount of degradation of neuronal mitochondria (Fig 2B).

327 Furthermore, similarly to the murine AD astrocytes, the iPSC-astrocytes derived from a symptomatic AD patient with the PSEN1 $\triangle E$ 9 mutation were observed to degrade significantly more neuronal mitochondria derived from iPSC-neurons compared to its isogenic, PSEN1 mutation corrected, control cells (mean difference 4,296 $\pm 1,156$, $\mathrm{p}=0.0005$ ). However, no significant difference in degradation of the neuronal mitochondria between the iPSCastrocytes derived from pre-symptomatic AD patient and its isogenic control cells was observed (Fig 2C). The degradation of neuronal mitochondria was the highest in murine astrocytes derived from 11 mo $5 \times \mathrm{FAD}$ mice (mean difference $18,56 \pm 4,478, \mathrm{p}=0.0001$ ) indicating an age-dependent alteration to transmitophagy in $\mathrm{AD}$ astrocytes. 
bioRxiv preprint doi: https://doi.org/10.1101/2021.09.15.460570; this version posted September 17, 2021. The copyright holder for this preprint (which was not certified by peer review) is the author/funder, who has granted bioRxiv a license to display the preprint in perpetuity. It is made available under aCC-BY-NC-ND 4.0 International license.

A

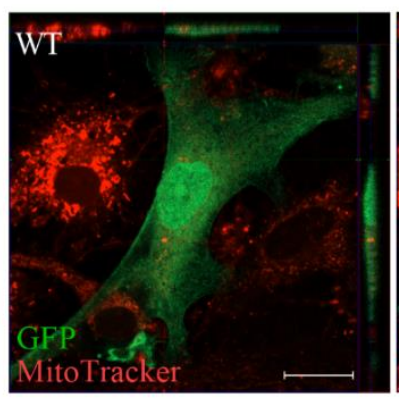

B

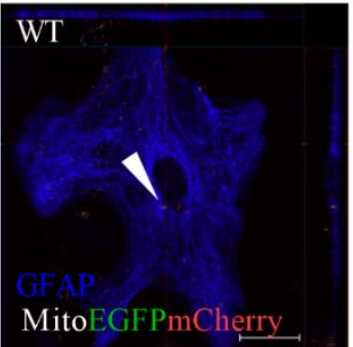

D
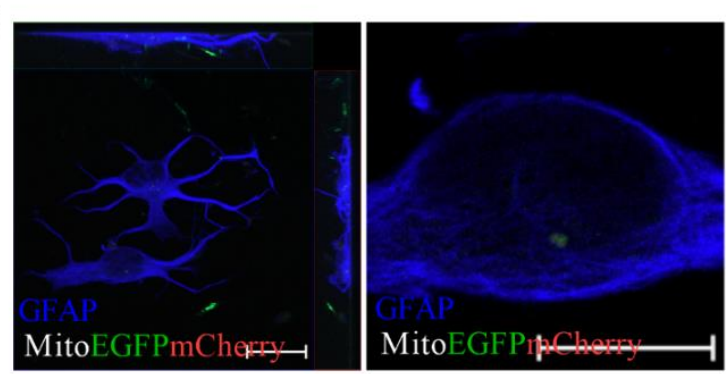

$\mathbf{F}$
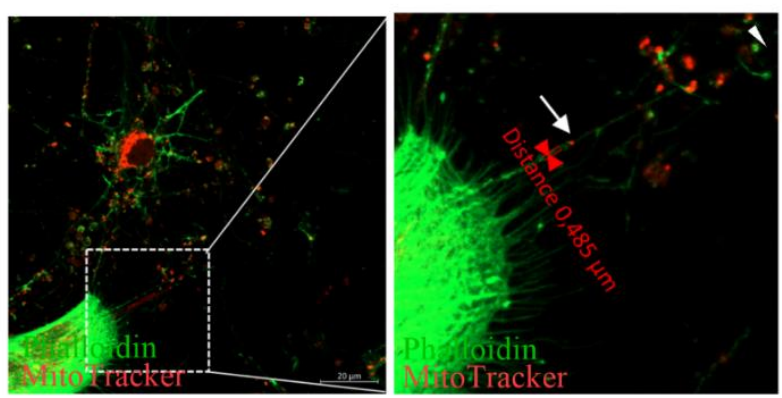

G

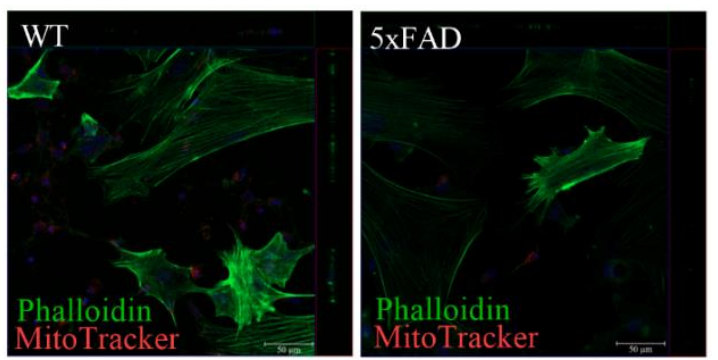

untreated

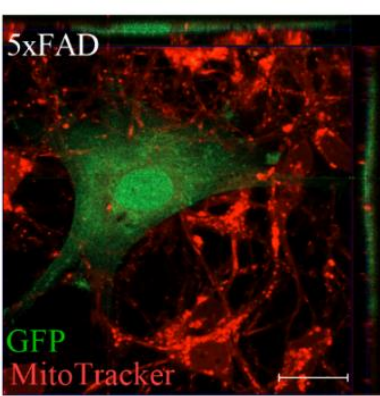

C

INTERNALIZATION: Murine astrocytes

(Neuronal mitochondria / astrocyte) \pm SD

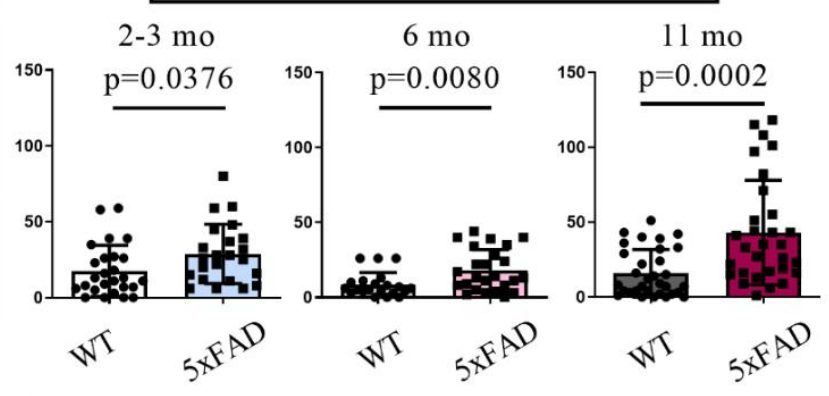

$\mathbf{E}$

INTERNALIZATION: Human astrocytes
(Neuronal mitochondria / astrocyte) \pm SD

Symptomatic Pre-symptomatic
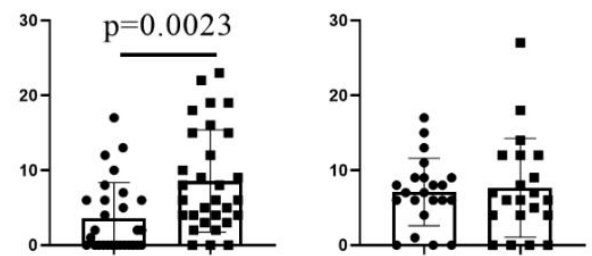

spespestents

be be bent

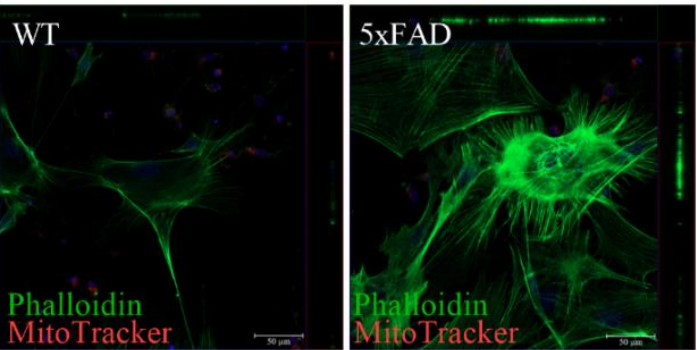

Neurons: $1 \mu \mathrm{M} \mathrm{H} 2 \mathrm{O} 2,2 \mathrm{~h}$ 

images from one z-plane showing neuronal mitochondria internalized by astrocytes in vitro in orthogonal view. E15 cortical neurons were labelled with MitoTracker CMXROS dye prior to co-culturing with adult astrocytes derived from 5 mo WT or 5xFAD mouse brains and expressing a lentiviral-GFP construct. Sacle bar $20 \mu \mathrm{m}$. (B) Example confocal images from one z-plane showing internalized neuronal mitochondria inside adult astrocytes in vitro in orthogonal view. Adult astrocytes were co-cultured with E15 neurons expressing a lentiviralmitoEGFPmCherry construct. Scale bar $20 \mu \mathrm{m}$. (C) Quantified amounts of internalized neuronal mitochondria in astrocytes harvested from 2-3 mo, 6 mo and 11 mo WT and 5xFAD mouse brain. Neuronal mitochondria were labeled with a lentiviral-mitoEGFPmCherry construct. Data is shown as all (mCherry only and mCherry + EGFP) fluorescence signal peaks/cell \pm SD. N=3 biologically independent replicates for each age-point. Each dot represents one single astrocyte imaged. Three biologically independent experiments were carried out for each agepoint. Unpaired two-tailed t test. (D) Example images showing degraded neuronal mitochondria inside astrocyte in iPSC-derived neuron- and astrocyte cultures. An internalized mitochondria is shown in maximum intensity projection of a z-stack (left hand side, scale bar $20 \mu \mathrm{m}$ ) and as a digital zoom-in from one z-stack plane for an iPSC-astrocyte with internalized neuronal mitochondria (right hand side, scale bar $10 \mu \mathrm{m}$ ).(E) Quantified amounts of neuronal mitochondria in iPSC-astrocytes derived from symptomatic and pre-symptomatic AD patients carrying PSEN1 mutation and their isogenic (mutation corrected) lines. N=22-30 analyzed astrocytes/ iPSC-line. spPSEN, symptomatic donor with clinical diagnosis for AD and PSEN1 $\triangle \mathrm{E} 9$ mutation. pPSEN, pre-symptomatic donor with PSEN1 $\triangle$ E9 mutation. SpISO, isogenic (PSEN1 mutation corrected) line for the symptomatic donor, pISO, isogenic (PSEN1 mutation corrected) line for the pre-symptomatic donor. Unpaired two-tailed t-test. (F) An example confocal image showing neuronal mitochondria traveling along a tunneling nanotube visualized with phalloidin staining. Neuronal mitochondria were labelled with MitoTracker CMXROS dye prior co-culturing with adult astrocytes harvested from 3 mo 5xFAD mouse brain. On the right-hand side an enlarged image of the boxed area. The white arrow heads point to examples of degraded neuronal mitochondria in astrocytes and white arrow 
of TNT-like structures were induced in astrocytes by treating the neurons with $1 \mu \mathrm{M} \mathrm{H} 2 \mathrm{O} 2$ for $2 \mathrm{~h}$ prior labeling the neuronal mitochondria with Mitotracker CMXROS dye and co-culturing them with astrocytes. TNT-like structures were visualized with phalloidin dye. Scale bar $50 \mu \mathrm{m}$. All graphs represent the mean \pm SD.

A

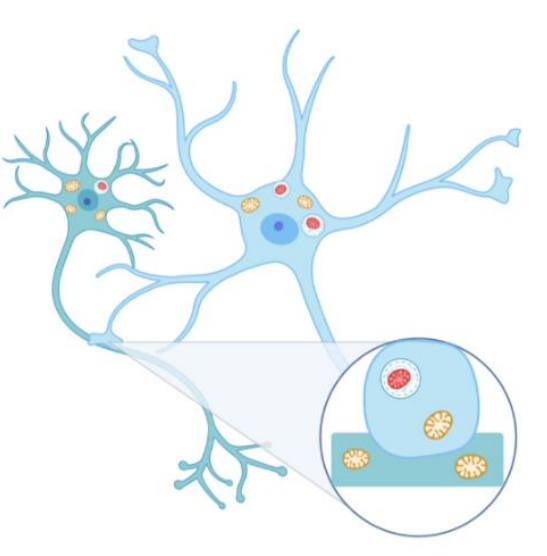

C

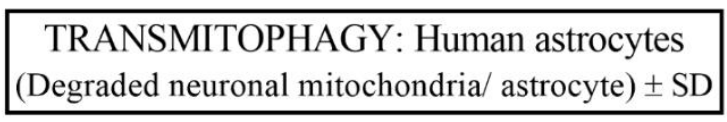

Symptomatic

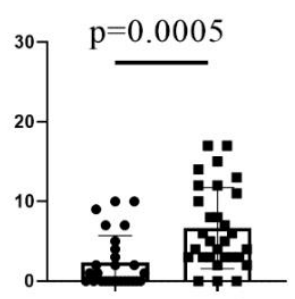

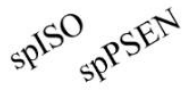

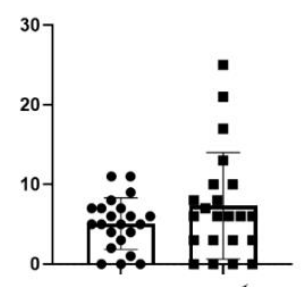

B

TRANSMITOPHAGY: Murine astrocytes
(Degraded neuronal mitochondria/ astrocyte) \pm SD

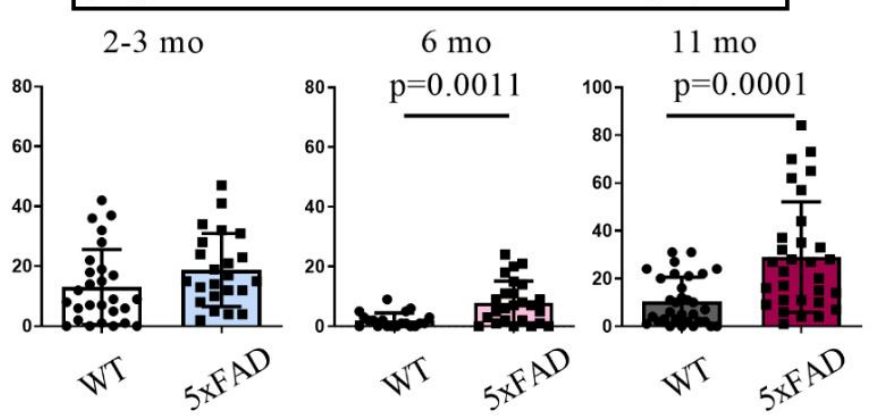

D
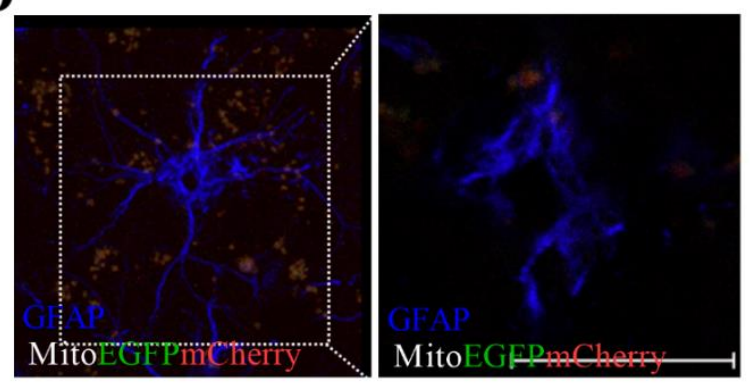

MitoEGFPmelpery

Figure 2. Astrocytes' ability to degrade neuronal mitochondria is altered in aging and in AD. (A) Graphical

illustration of the lentiviral-mitoEGFPmCherry construct. The synapsin promoter driven mitochondria-targeted reporter construct contains EGFP (green) and mCherry (red), which colocalize in mitochondria (yellow signal). Upon meeting the acidic environment of the lysosome, the EGFP signal is lost, resulting in red mCherry fluorescence, indicative of mitochondrial degradation. (B) Quantified amounts of degraded neuronal mitochondria in astrocytes harvested from 2-3 mo, 6 mo and 10-11 mo WT and 5xFAD mouse brains. Neuronal mitochondria 
374 peaks/cell \pm SD. N=3 biologically independent replicates for each age-point. Each dot represents one single

375

376

377

378

379

380

381

382

383

384

385

386

387

388

389

390

391

392

393

394

395

396

397

398

astrocyte imaged. Three biologically independent experiments were carried out for each age-point. Unpaired two-

tailed t test. (C) Quantified amounts of degraded neuronal mitochondria in iPSC-astrocytes derived from symptomatic and pre-symptomatic AD patients carrying PSEN1 mutation and their isogenic (mutation corrected) lines. N=22-30 analyzed astrocytes/ iPSC-line. spPSEN, symptomatic donor with clinical diagnosis for AD and PSEN1 $\triangle$ E9 mutation. pPSEN, pre-symptomatic donor with PSEN1 $\triangle$ E9 mutation. SpISO, isogenic (PSEN1 mutation corrected) line for the symptomatic donor, pISO, isogenic (PSEN1 mutation corrected) line for the presymptomatic donor. Unpaired two-tailed t-test. (D) Maximum intensity projection of a z-stack and orthogonal view from one z-stack plane (right hand side) visualizing degraded neuronal mitochondria inside an astrocyte in the hippocampus of a 6 mo 5xFAD mouse and a digital zoom-in from one z-stack plane with internalized neuronal mitochondria (right hand side, scale bar $20 \mu \mathrm{m}$ ).

\subsection{Aged 5xFAD mice display a shift in immunoreactivity for $\mathrm{S100a4}$ from neurons to astrocytes}

The concentration gradient of the $\mathrm{S} 100 \mathrm{a} 4$ protein between neurons and astrocytes has been shown to determine the direction of TNT formation, which serve as potential transcellular highways for mitochondrial transfer between these cell types (Sun et al., 2012). To determine its involvement in the mitochondrial transfer from neurons to astrocytes, we assessed levels of S100a4 by immunohistochemistry in 5xFAD mouse brain sections. The immunoreactivity of S100a4 was not significantly reduced in the areas highly enriched with hippocampal neurons of 12 mo 5xFAD mice when compared to age-matched WT mice, although there was seen a trend for reduced immunoreactivity in areas enriched with neurons the images of the anti-S100a4 immunostained brain sections (data not shown). On the other hand, in the fiber tract area, populated primarily by astrocytes, a significant increase (mean difference above CA1 $4.300 \pm 0.4966, \mathrm{p}<0.0001$ and mean difference above CA3 $2.967 \pm 0.3850, \mathrm{p}<0.0001$ ) in immunoreactivity for S100a4 was observed in 12 mo 5xFAD mice (Fig 3A). In addition, in 5xFAD sections the S100a4 staining was observed close to areas stained with anti-amyloid $\beta$ antibody (Fig 3A).

We performed further an ELISA assay for S100a4 from cell lysates of adult astrocytes derived from 5-6 mo and 11-12 mo WT and 5xFAD mice (Supplementary figure 1). The results indicate an increase of intracellular S100a4 
399 in astrocytes harvested from 12 mo 5xFAD mice compared to the other study groups, similarly as immunostaining

400 for S100a4 was observed to be increased in 12mo 5xFAD mouse brain in astrocyte-rich areas.

401 To assess the S100a4 immunoreactivity also in the human brain, we immunostained post mortem human brain 402 sections with both anti-S100a4 antibody and Nissl staining. Based on these example images it seems that in the 403 human brain the AD neurons have less S100A4 in the hippocampal layer, where the neurons are visualized with 404 Nissl-staining. However, in the cortex the AD brain seemed to have more positive immunostaining for S100A4 in 405 other cell types besides neurons (Fig 3B). These data are in line with our findings in the 5xFAD mice for the glial406 like cells. 
bioRxiv preprint doi: https://doi.org/10.1101/2021.09.15.460570; this version posted September 17, 2021. The copyright holder for this preprint (which was not certified by peer review) is the author/funder, who has granted bioRxiv a license to display the preprint in perpetuity. It is made available under aCC-BY-NC-ND 4.0 International license.

A

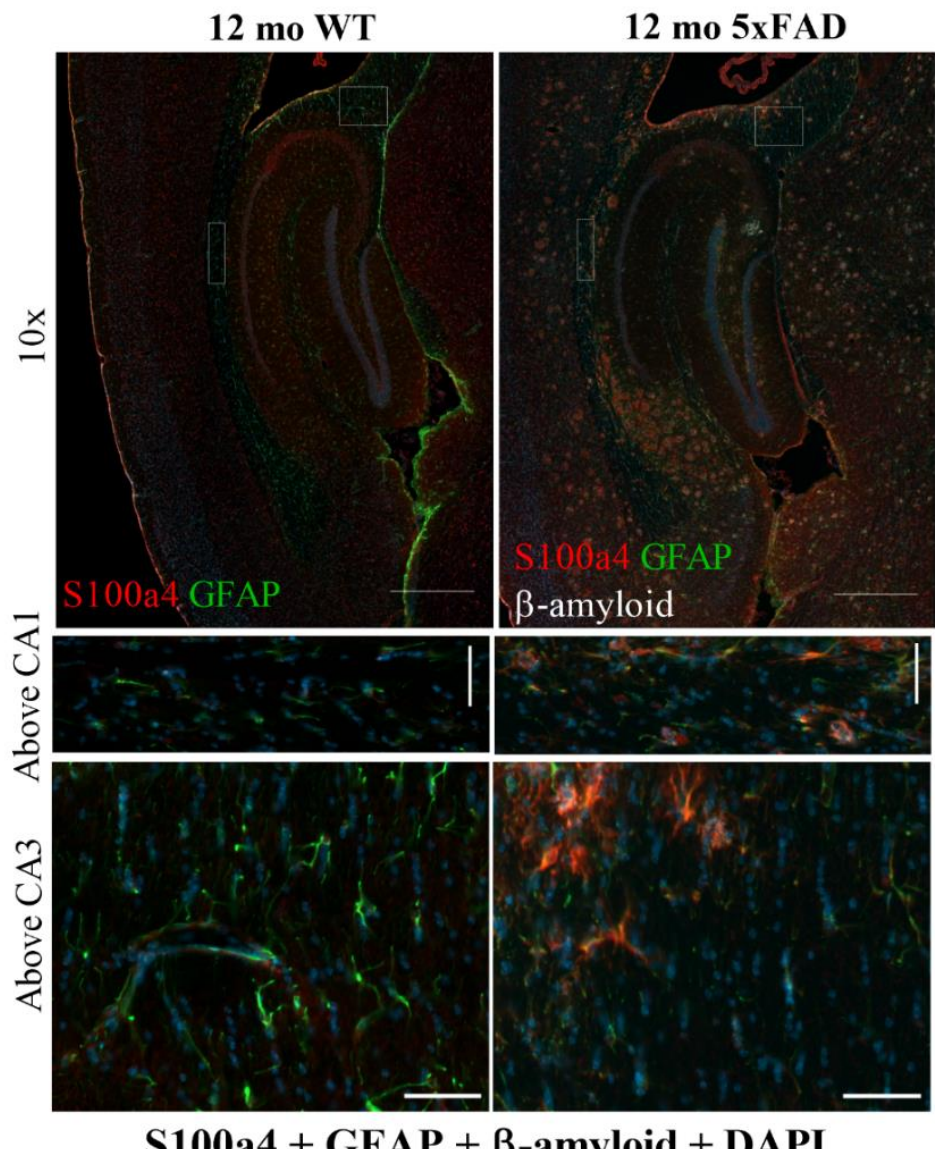

Astrocytes

Fiber tract above CA1

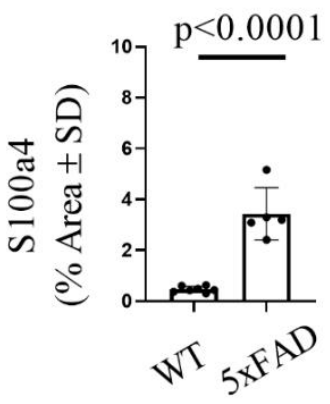

Fiber tract above CA3

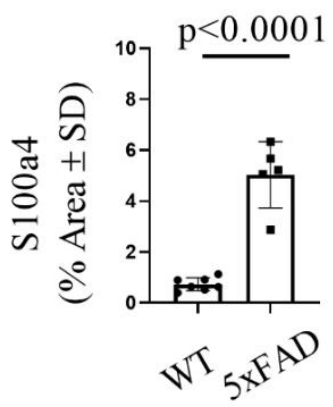

B

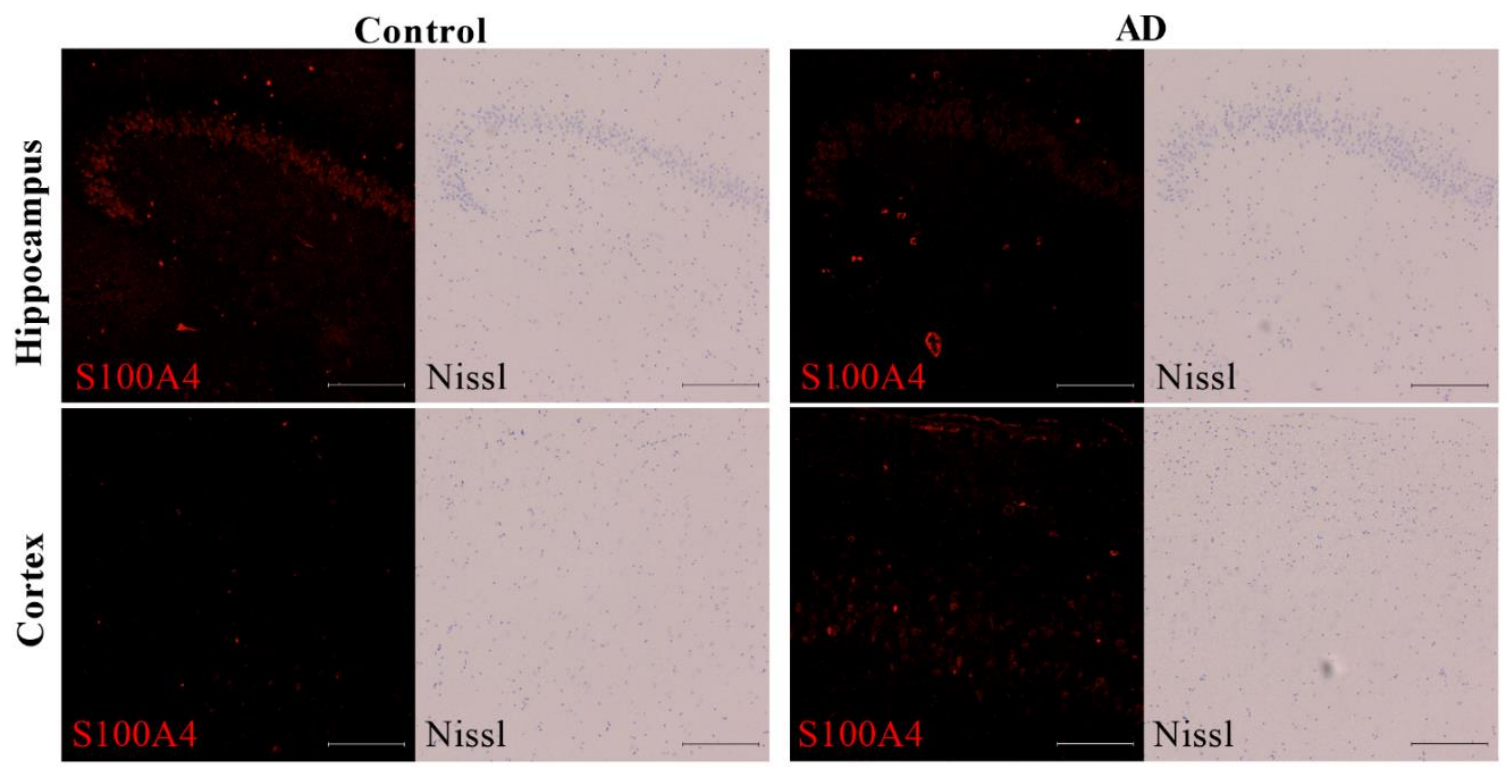


Figure 3. S100a4 is increased in aged 5xFAD astrocytes in vivo. (A) The immunoreactivity of S100a4 was by

histochemical staining from cryosections of 12 mo 5xFAD and WT brain sections in cells resembling astrocytes

by morphology in the fiber tract region above CA1 and CA3 neuronal layers. Quantitative data is presented as

immunoreactive area for $\mathrm{S} 100 \mathrm{a} 4, \% \pm \mathrm{SD} . \mathrm{N}=7 \mathrm{WT}$ mice and $\mathrm{N}=4$ 5xFAD mice. Example images with $10 \mathrm{x}$ objective (scale bar $500 \mu \mathrm{m}$ ) and enlargement of boxed areas (scale bar $50 \mu \mathrm{m}$ ) showing the difference between genotypes. Anti-S100a4 staining is shown as red, anti-GFAP as green, DAPI as blue and anti- amyloid $\beta$ with

white pseudo color. Unpaired two-tailed t test. (B) The immunoreactivity for S100a4 in neuron-enriched areas was assessed by immunohistochemical staining in paraffin sections of human brain posterior hippocampus. The location of neuronal cells was visualized with Nissl staining. Example images were taken with 10x objective. Scale bar $200 \mu \mathrm{m}$.

\subsection{Mitochondrial functions of astrocytes, astrocyte reactivity and phagocytosis are unaltered upon aging} and AD

The observation of increased internalization and degradation of neuronal mitochondria by aged 5xFAD astrocytes led us to investigate the functionality and health of the astrocytes' own mitochondria. We first assessed cytochrome c oxidase (mitochondrial electron transport chain complex IV) activity in astrocytes in WT and 5xFAD mouse brains by histochemistry. The cytochrome c oxidase activity appeared to be altered specifically around betaamyloid plaques in aged 5xFAD brains (Suppl. Fig. 2A), indicating that the mitochondrial function of a subset of astrocytes may be altered in vivo. However, we did not observe a difference in the basal respiration rate between age-matched 5xFAD and WT astrocytes (Suppl. Fig 2B). Upon comparing the 5xFAD astrocytes harvested from 5-6 mo mice to those extracted from 10-12 mo 5xFAD mice, the 5xFAD astrocytes from 5-6 mo mice was observed to have significantly higher basal respiration rate. Furthermore, levels of intracellular ATP were not altered when comparing WT and 5xFAD astrocytes (Suppl. Fig 2C) and live cell imaging of the mitochondrial membrane potential of astrocytes did not reveal alteration in 5xFAD astrocytes (Suppl. Fig. 2D). The absence of mitochondrial alterations in 5xFAD astrocytes was further supported by measurement of the mitochondrial content 
during aging and in 5xFAD cells (Suppl. Fig 2E). To rule out the possibility of increased levels of oxidative stress in the aged 5xFAD astrocytes, the gene expression levels of the antioxidant-response genes heme oxygenase-1 (Hmoxl) and $\mathrm{NAD}(\mathrm{P}) \mathrm{H}$ : quinone oxidoreductase (Nqol) were assessed by qPCR. However, no significant difference between WT and 5xFAD astrocytes was observed (data not shown).

In rodent brains, the astrocytes have been reported to manifest as the reactive sub-phenotype, termed $\mathrm{A} 1$, in $\mathrm{AD}$ and normal aging (Clarke et al., 2018; Liddelow et al., 2017). To determine how aging and AD affect key astrocytic functions besides mitochondrial internalization and degradation, we assessed their reactive phenotype and phagocytic ability. The expression level of the A1 marker gene Serping1 was observed to be increased and while the other A1 marker gene Srgn expression was reduced in astrocytes isolated from the adult 5xFAD mouse brains, indicating that the FAD mutations alter the phenotype of astrocytes (Suppl. Fig. 3A). The expression of genes encoding phagocytosis-related proteins was unchanged in $\mathrm{AD}$ and during aging of astrocytes except for upregulation of $\mathrm{Megf10}(\log 2 \mathrm{FC} 1.0689, \mathrm{p}<0.045)$ in $5 \mathrm{xFAD}$ astrocytes at 2-3 mo age compared to the astrocytes harvested from age-matched WT mice (Suppl. Fig. 3B). Furthermore, no difference was observed in phagocytosis of pHrodo Zymosan A bioparticles between the WT and 5xFAD astrocytes at all ages, indicating that the phagocytic capacity of astrocytes remains unaltered (Suppl. Fig 3C). The 5xFAD astrocytes were not observed to increase secretion of inflammatory cytokines compared to WT (Supplementary Tables 2 and 3).

\subsection{Astrocyte-mediated neuronal support is altered in AD}

We next assessed whether the ability of astrocytes to support neuronal functions is altered upon aging and/or AD, possibly thereby elucidating why the internalization and degradation of neuronal mitochondria is increased in the aged 5xFAD astrocytes. Based on live cell imaging with rhodamine 123 dye, co-culturing primary neurons with 5xFAD astrocytes from aged mice altered the mitochondrial membrane potential (MMP) of neurons (Fig 4A). When the neurons were co-cultured with 5xFAD astrocytes and treated with glutamate (mimicking the glutamate excitotoxicity in $\mathrm{AD}$ ) neurons co-cultured with 11-12 mo 5xFAD astrocytes exhibited significantly higher MMP, compared to that with 5-6 mo mice. The 11-12 mo WT astrocytes were not observed to induce similar alterations in the neuronal MMP under vehicle or glutamate treatment. Prolonged high MMP has been reported to increased 
458 production of mitochondrial reactive oxygen species (ROS) and up-regulate autophagy, especially in neurons 459 treated with glutamate (Kumari et al., 2012). Interestingly, both the WT and 5xFAD astrocytes isolated from 1146012 mo mice were incapable of buffering neuronal viability from the effects of glutamate exposure (Fig 4B). 461 However, treating the co-cultures of neurons and astrocytes isolated from 5-6 mo mice WT or 5xFAD mice with 462 glutamate had no effect on the viability of the neurons, suggesting an age-dependent reduction in astrocyte 463 functions relating to neuronal support. Furthermore, the 11-12 mo 5xFAD astrocytes secreted reduced levels of 464 anti-inflammatory interleukin 10 (IL-10) and interferon gamma (IFN- $\gamma$ ) in comparison to age-matched WT 465 astrocytes (Supplementary Tables 2 and 3).

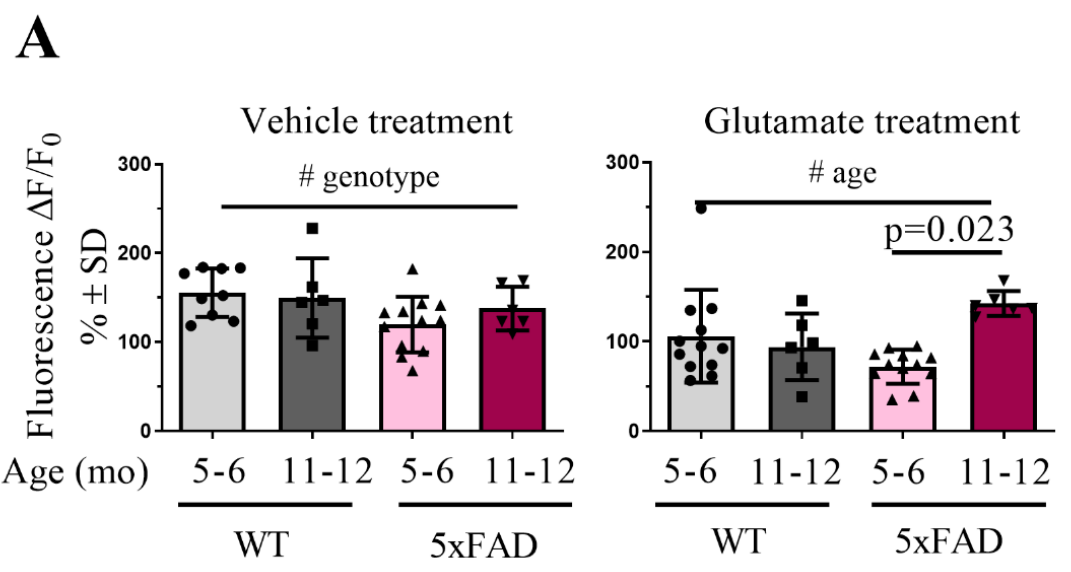

\section{B}
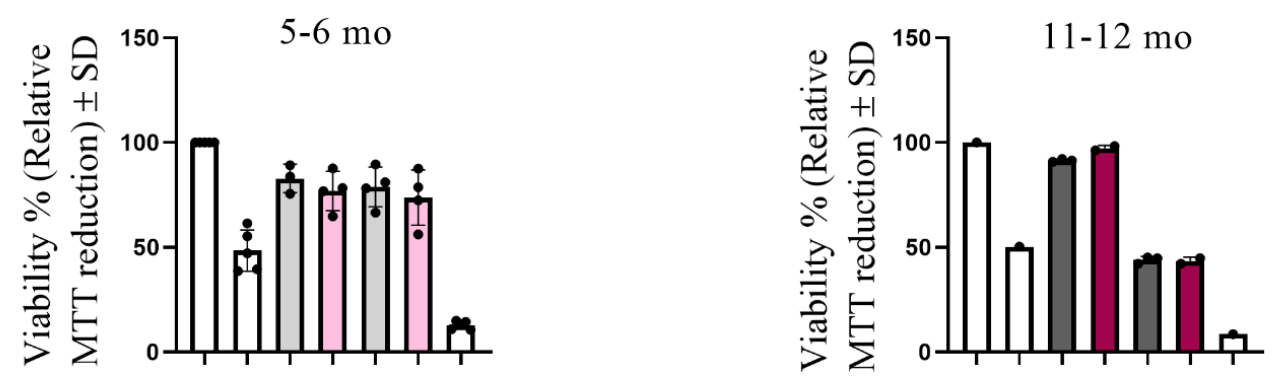

- + - - + - Glutamate

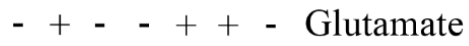

- - + + - - WT astrocytes

- - + + - - WT astrocytes

- - + - + - 5xFAD astrocytes

- - + - + - 5xFAD astrocytes

- - - - + Triton X-100 (lysed)

- - - - + Triton X-100 (lysed) 
Figure 4. Astrocytes' ability to support neuronal functions is altered in AD. (A) Mitochondrial membrane imaging with rhodamine 123 dye. The astrocytes were cultured on inserts on top of the neurons for 2 days prior the experiment and co-cultures were treated with $250 \mu \mathrm{M}$ glutamate for $24 \mathrm{~h}$ before the live cell imaging. The bar graph shows the cells response to $\mathrm{FCCP}$ as $\Delta \mathrm{F} / \mathrm{F}_{0}$ (normalized to baseline), $\% \pm \mathrm{SD}$. For neurons co-cultured with astrocytes harvested from 5-6 mo mice the data is shown as an average of 3-4 individual experiments with 3 technical replicates in each $(n=3-4$ mice per group in total). For astrocytes harvested from 10-12 mo mice, data shown as an average of 2 experiments with 3 technical replicates in each ( $\mathrm{N}=2$ mice per group in total). All graphs represent the mean \pm SD. Two-way ANOVA genotype effect $p=0.0495$ for vehicle treated cultures and age-effect for the glutamate treated cultures $\mathrm{p}=0.0274$. (B) The viability of the neurons was assessed with the MTT assay. The histograms show relative MTT reduction in neurons \pm SD when neurons were co-cultured with or without astrocytes isolated from 5-6 mo or 11-12 mo) WT or 5xFAD mice for 2 days prior the experiment. For $24 \mathrm{~h}$ before the MTT assay all the cultures were treated with $250 \mu \mathrm{M}$ glutamate or vehicle solution. Data is shown as an average of 3-5 individual experiments with 5-6 mo astrocytes and 1 experiment with 11-12 mo astrocytes ( $\mathrm{N}=3-4$ mice for astrocytes isolated from 5-6 mo mice, $\mathrm{N}=2-3$ mice for astrocytes isolated from 11-12 mo mice). One-way ANOVA.

\subsection{Protein levels for mitophagy-inducer Ambra1 are significantly increased in aged 5xFAD astrocytes}

To decipher whether autophagy-related processes are responsible for the observed transmitophagy alteration in aged $\mathrm{AD}$ astrocytes we assessed protein levels of common autophagy markers by Western blot. Immunoblotting against mitophagy- and autophagy-related proteins, p62 and LC3b, did not reveal significant changes in in astrocytes during aging or in $\mathrm{AD}$ (Fig. 5B). However, a two-fold increase in the protein level of Ambra1, a mitophagy inducer, was observed in aged (11-12 mo) 5xFAD astrocytes compared to WT astrocytes (Fig. 5A). 

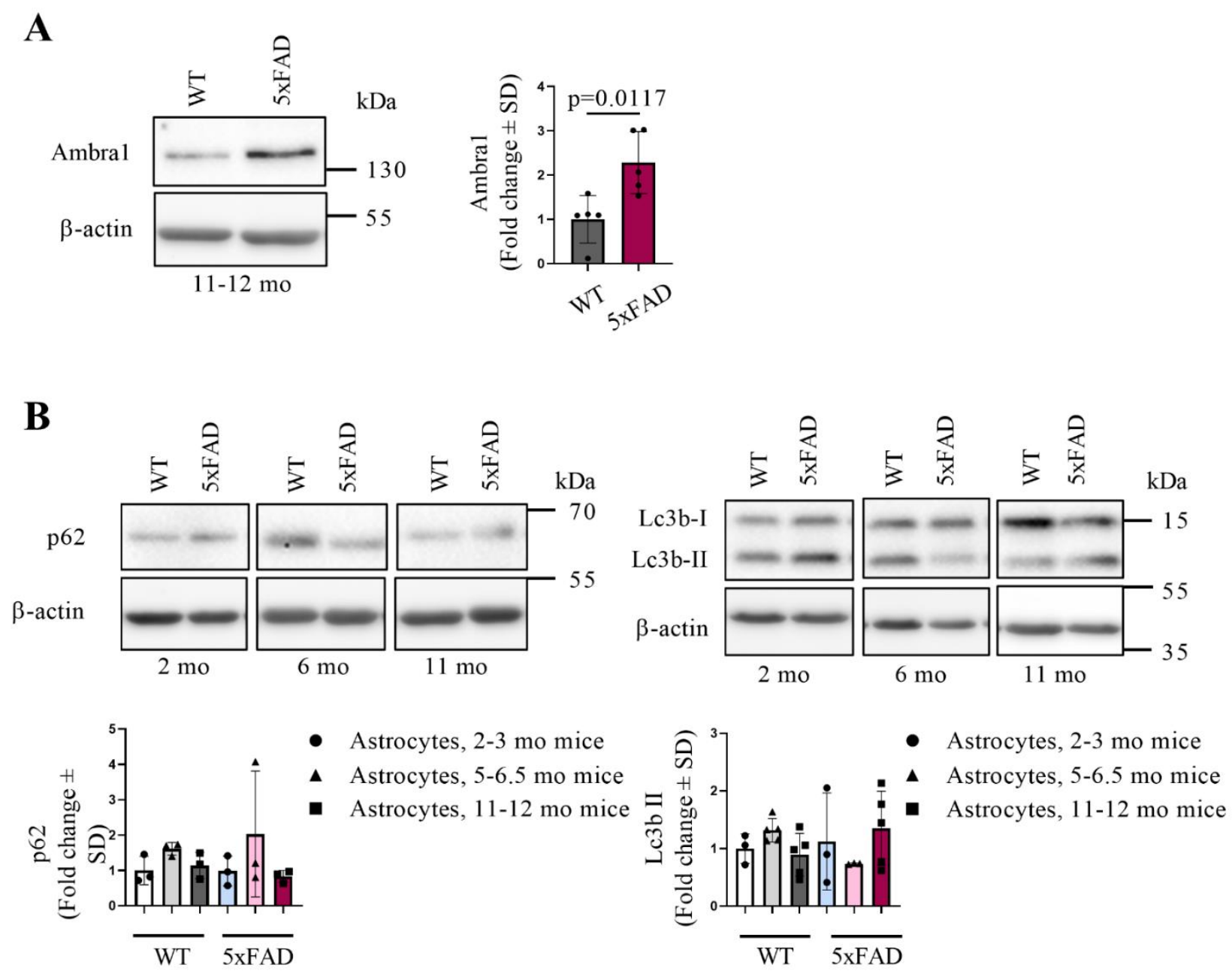

Figure 5. Expression of mitophagy-inducer Ambra1 is increased selectively in aged AD astrocytes. (A) The

492 relative protein level of Ambra1 was assessed by Western blotting in adult astrocytes. N=3-5 mice per group.

493 Unpaired two-tailed t test. (B) The relative protein levels of p62 and LC3B were assessed by Western blotting in 494 adult astrocytes. $\mathrm{N}=3-5$ mice per group. The ratio of protein expression was determined by normalizing the level 495 of each protein to that of $\beta$-actin and the normalized value ratio to that of the value of $2-3$ mo WT mice. In bar plots one dot represent astrocytes harvested from a single mouse.

\section{Discussion}

Many previously accepted facts about mitochondria have been recently re-evaluated, including the assumptions that each cell degrades its own mitochondria (Davis et al., 2014), and that mitochondria and their DNA are 
500

501

502

503

504

505

506

507

508

509

510

511

512

513

514

515

516

517

518

519

520

521

522

523

524

exclusively maternally inherited (Luo et al., 2018). These new findings have provided more on the importance of this organelle for previously unappreciated functions. The results presented herein demonstrate for the first time both in mouse and human that mitochondrial transfer occurs between neurons and AD-affected astrocytes, and that $\mathrm{AD}$ astrocytes degrade mitochondria derived from neurons. Moreover, mitochondrial transcellular movement and transmitophagy are impaired in AD.

Experiments with astrocytes treated with $\mathrm{A} \beta$ peptides have reported the induction of multiple alterations in astrocytic mitochondrial function (Abramov et al., 2004; Sarkar et al., 2014), but there are not many reports of mitochondrial functions of astrocytes isolated from mice modeling AD. Mitochondrial fractions of neonatal 5xFAD astrocytes have been shown to contain altered levels of metabolites and enzymatic activity related to the glycolytic pathway and TCA cycle. Similar changes were observed in WT astrocytes after exposure to oligomeric A $\beta$. (van Gijsel-Bonnello et al., 2017) One could think that defects in mitochondrial health of AD-affected astrocytes leads to increased internalization of neuronal mitochondria, for example due to an attempt to correct an energy deficit. However, our studies on the mitochondrial functions of astrocytes isolated from adult 5xFAD mouse brains did not reveal significant differences when compared to age-matched WT astrocytes in any of the assays we performed.

TNTs, thin elongations of the cell membrane consisting of F-actin, serve as highways for organelle transfer, including mitochondria, between cells (Rustom et al., 2004; Spees et al., 2006) Our data demonstrates the formation of TNT-like structures between neurons and astrocytes, and suggests they may mediate the transfer of mitochondria between these cell types. Although not assessed in the current paper, previous studies have shown that astrocytes from neonatal APP/PS1 mice form more TNTs than their WT counterparts (Sun et al., 2012) This finding supports our results and possibly explains, at least in part, our finding of increased mitochondrial transfer that occurs in aged 5xFAD astrocytes. The formation of TNTs between cells is driven by a concentration gradient of the protein S100a4, cleaved by caspase-3. The cell initiating the TNT formation has reduced levels of S100a4 compared to the recipient cells with higher S100a4 concentration (Sun et al., 2012). Interestingly, Yao et al. have observed increased caspase-3 activity in U87 astrocyte-like cells after treating the cells with A $\beta$ (Yao et al., 2018). 
525 The presence of $\mathrm{AD}$ pathology manifested as increased levels of brain $\mathrm{A} \beta_{1-42}$ could be speculated to activate 526 caspase-3, leading to reduced levels of S100a4 in affected cells. Our results suggest that S100a4 levels are reduced 527 in aged 5xFAD hippocampal neurons. On the other hand, astrocytes in the aged 5xFAD brain and 11-12 mo 5xFAD 528 astrocytes cultured in vitro display increased S100a4. These findings imply that the aged 5xFAD astrocytes 529 function as recipient cells for TNT-like structures formed by neurons, since the S100a4 protein gradient formed 530 between these cell types would allow the transfer of mitochondria along TNT-like structures. This is in line with 531 our observation of increased internalization of neuronal mitochondria by 5xFAD astrocytes over the WT 532 astrocytes. Previously studies have also shown astrocytic S100a4 to be increased after head trauma or astrocytes 533 under stress (Dmytriyeva et al., 2012; Kozlova and Lukanidin, 2002). The protein has been shown to be 534 neuroprotective, acting partly via binding to the IL-10 receptor and via JAK/STAT3 pathway (Dmytriyeva et al., 535 2012). In addition, the authors suggested that S100a4 may be potent for activating the IL10R/Akt pathway. In 536 other studies IL-6, IL-8 and especially IL-7 have been reported to function via JAK/STAT3 pathway and stimulate 537 secretion of S100a4 from human chondrocytes (Yammani et al., 2009). In addition, when stimulated, adult 5xFAD astrocytes have been reported to secrete threefold more IL-6 compared to WT astrocytes (Iram et al., 2016). We did not observe differences in levels of secretion of IL-6 from unstimulated aged WT and 5xFAD astrocytes. Our result of increased S100a4 in astrocytes of the 12 mo 5xFAD mouse brain together with reduced secretion of antiinflammatory IL-10 may, however, imply the involvement of the S100a4-IL-10 axis in AD astrocytes and in mitochondrial transfer between neurons and astrocytes.

543 Transmitophagy has been shown to occur in rodent astrocytes in vivo adjacent to the optic nerve head (Davis et 544 al., 2014) and between dopaminergic neurons and their neighboring in the context of Parkinson's disease (Morales 545 et al., 2020). Our data are the first to demonstrate transmitophagy of neuron-derived mitochondria by human iPSC546 derived astrocytes, and alterations to this process upon aging in AD astrocytes. Our data also supports the existence 547 of transmitophagy both in vitro and in vivo. Altered transmitophagy was not associated with astrocytic phagocytosis capacity and basic mitochondrial functions, yet the cytochrome c oxidase activity appeared to be 
significantly increased Ambra1 levels when compared to their WT counterparts. Existing literature supports the hypothesis of altered COX-activity in 5xFAD mice brains. Devi and Ohno (2012) have shown increased release of mitochondrial cytochrome $\mathrm{c}$ to cell cytosol in 5xFAD mice brains compared to the WT brains, regardless of the mouse age. In addition, the authors have described a trend $(\mathrm{p}=0.06)$ towards reduced COX activity in 12 monthold 5xFAD mouse brains (Devi and Ohno, 2012). Mitophagy induction via Ambra1 by its binding to LC3 is known to occur independently of the classical mitophagy-related protein Parkin (Strappazzon et al., 2015). Levels of p62 remained unaltered but the levels of LC3bII seemed to be slightly increased in the aged (11-12 mo) 5xFAD astrocytes. This suggests that unlike in synaptosomal (neuronal) mitochondria, in which aged 5xFAD cells display increased LC3bII and Parkin translocation (Wang et al., 2016), increased mitophagy in 5xFAD astrocytes is the result of up-regulated Ambra1 and its action together with LC3.

A key finding in this study is that a large amount of neuronal mitochondria are internalized and degraded by astrocytes in co-cultures. This finding agrees with a previous study reporting that degradation of large amounts of axonal mitochondria by glial cells in the optic nerve head (Davis et al., 2014). Notably, we also found mitochondria with both EGFP and mCherry signals inside the astrocytes, denoting mitochondria not yet present in an environment with a low $\mathrm{pH}$ suitable for degradation. Previous data indicated that the degrading neuronal mitochondria were found in astrocytes expressing high levels of marker for phagocytosis, Mac2 (Davis et al., 2014). On the other hand, reactive A1 type of astrocytes have been reported to be to present in AD-affected brains and during normal aging, showing reduced ability for neuronal support (Clarke et al., 2018; Liddelow et al., 2017). For example, in the article by Liddelow et al., astrocytes isolated from neonatal mice and induced to the A1 type by reactive microglia displayed reduced synapse engulfment and reduced gene expression of the phagocytosis related genes Megf10 and Mertk. However, our data did not indicate alterations to phagocytosis by the aged 5xFAD astrocytes, suggesting that the phagocytosis process may not be responsible for the observed effects in transmitophagy and internalization of neuronal mitochondria. 
575

576

577

578

579

580

581

582

583

584

585

586

587

588

589

590

591

592

593

594

595

596

597

as an energy source in cultured astrocytes (Yan et al., 2017). Our results indicated that glutamate treatment reduced neuronal viability when neurons were co-cultured with aged (11-12 mo) astrocytes despite the genotype of the astrocytes. However, the aged 5xFAD astrocytes failed to prevent the effects of glutamate excitotoxicity on neuronal MMP. The inability of neuronal mitochondria to reduce MMP upon glutamate exposure in a neuronastrocyte co-culture indicates a dysfunctional response in the aged astrocytes, resulting in increased stress and loss of quality, thus direction for degradation including transmitophagy. Recently it was reported that only those retinal ganglion cells that were already damaged by a previous insult were prone to cellular death induced by neurotoxic astrocytes (Guttenplan et al., 2020). This is in line with our observations of the effects of aged and especially aged $5 \mathrm{xFAD}$ astrocytes on the neuronal health of glutamate treated neurons.

In the past, researchers have heavily relied on conducting experiments with astrocytes isolated from neonatal mice. In this study we utilized astrocytes isolated from the brains of adult mice at various age points and report significant age- and genotype-related differences in the performed assays, emphasizing the importance of using cells derived from aged animals especially when studying age-related diseases. Transmitophagy was also observed in human iPSC-derived astrocytes and in vivo in the mouse hippocampi. Further studies should focus on developing means to study transmitophagy in vivo in the human brain and to assess whether the observed alterations represent an early indication of AD-related pathology. Taken together, our results along with the existing data highlights the importance of mitochondrial traffic between neurons and astrocytes and demonstrates AD-induced alterations to astrocytic functions.

\section{Acknowledgements}

We thank Mrs. Mirka Tikkanen, Ms. Laila Kaskela and Ms. Anna Palmgren for their expert technical assistance. The study was supported by Academy of Finland, Sigrid Juselius Foundation and University of Eastern Finland, and carried out with the support of UEF Cell and Tissue Imaging Unit, University of Eastern Finland, Finland. The authors thank Biocenter Kuopio Viral Gene Transfer service for providing lentiviral vectors for GFP.

\section{Disclosure}


599 The authors have no actual or potential conflicts of interest.

600 Data availability statement

601 Data available on reasonable request from the corresponding author.

602 References

603

604

605

606

607

608

609

610

611

612

613

614

615

616

617

618

619

620

621
Abramov, A. Y., Canevari, L., \& Duchen, M. R. $\beta$-Amyloid Peptides Induce Mitochondrial Dysfunction and Oxidative Stress in Astrocytes and Death of Neurons through Activation of NADPH Oxidase. J Neurosci. 2004; 24: 565-75.

Clarke, L. E., Liddelow, S. A., Chakraborty, C., Münch, A. E., Heiman, M., \& Barres, B. A. Normal aging induces A1-like astrocyte reactivity. Proc Natl Acad Sci U S A 2018; 115 : E1896-E05.

Davis, C. H. O., Kim, K. Y., Bushong, E. A., Mills, E. A., Boassa, D., Shih, T., ... MarshArmstrong, N. Transcellular degradation of axonal mitochondria. Proc Natl Acad Sci U S A 2014; 111: 9633-38.

Devi, L., Ohno M. Mitochondrial dysfunction and accumulation of the $\beta$-secretase-cleaved Cterminal fragment of APP in Alzheimer's disease transgenic mice. Neurobiol Dis. 2012; $45: 417-24$.

Dmytriyeva, O., Pankratova, S., Owczarek, S., Sonn, K., Soroka, V., Ridley, C. M., Marsolais A., Lopez-Hoyos M., Ambartsumian N., Lukanidin E., Bock E., Berezin V., Kiryushko, D. The metastasis-promoting S100A4 protein confers neuroprotection in brain injury. Nat Commun. 2012; 3: 1197.

English, K., Shepherd, A., Uzor, N. E., Trinh, R., Kavelaars, A., Heijnen, C. J. Astrocytes rescue neuronal health after cisplatin treatment through mitochondrial transfer. Acta Neuropathol Commun. 2020; 8: 36. 
Fang, E. F., Hou, Y., Palikaras, K., Adriaanse, B. A., Kerr, J. S., Yang, B., Lautrup S., M.M. Hasan-Olive Caponio D., Dan X., Rocktäschel P., Croteau D.L., Akbari M., Greig N.H., Fladby T., Nilsen H., Cader M.Z., Mattson M.P., Tavernarakis N., Bohr, V. A. Mitophagy inhibits amyloid- $\beta$ and tau pathology and reverses cognitive deficits in models of Alzheimer's disease. Nat Neurosci. 2019; 22: 401-12.

Foti, S. C., Hargreaves, I., Carrington, S., Kiely, A. P., Houlden, H., Holton, J. L. Cerebral mitochondrial electron transport chain dysfunction in multiple system atrophy and Parkinson's disease. Sci Rep. 2019, 9, 6559.

Guttenplan, K. A., Stafford, B. K., El-Danaf, R. N., Adler D.I., Münch A.E., Weigel, M. K., Huberman, A. D., Liddelow, S. A. Neurotoxic Reactive Astrocytes Drive Neuronal Death after Retinal Injury. Cell Rep. 2020; 31: 107776.

Hayakawa, K., Esposito, E., Wang, X., Terasaki, Y., Liu, Y., Xing, C., Ji X., Lo, E. H. Transfer of mitochondria from astrocytes to neurons after stroke. Nature 2016; 535: 551-55.

Hong, Y., Liu, Y., Zhang, G., Wu, H., Hou, Y. Progesterone suppresses A 342 -induced neuroinflammation by enhancing autophagy in astrocytes. Int Immunopharmacol. 2018; 54 : $336-43$.

Iram, T., Trudler, D., Kain, D., Kanner, S., Galron, R., Vassar, R., Barzilai A., Blinder P., Fishelson Z., Frenkel, D. Astrocytes from old Alzheimer's disease mice are impaired in A $\beta$ uptake and in neuroprotection. Neurobiol Dis. 2016; 96: 84-94.

Kanninen, K., Heikkinen, R., Malm, T., Rolova, T., Kuhmonen, S., Leinonen, H., Ylä-Herttuala S., Tanila H., Levonen A-L., Koistinaho M., Koistinaho, J. Intrahippocampal injection of a lentiviral vector expressing Nrf2 improves spatial learning in a mouse model of Alzheimer's disease. Proc Natl Acad Sci U S A 2019; 106: 16505-10. 
645

646

647

648

649

650

651

652

653

654

655

656

657

658

659

660

661

662

663

664

665

666

667

Konttinen H., Gureviciene I., Oksanen M., Grubman A., Loppi S., Huuskonen M.T., Korhonen P., Lampinen R., Keuters M., Belaya I., Tanila H., Kanninen K.M., Goldsteins G., Landreth G., Koistinaho J., Malm T. PPAR $\beta / \delta$-agonist GW0742 ameliorates dysfunction in fatty acid oxidation in PSEN1 $\triangle$ E9 astrocytes. Glia, 2019;67:146-59.

Kozlova, E. N., Lukanidin, E. Mts1 protein expression in the central nervous system after injury. Glia 2002; 37: 337-48.

Kumari, S., Mehta, S. L., Li, P. A. Glutamate induces mitochondrial dynamic imbalance and autophagy activation: Preventive effects of selenium. PLoS ONE 2012; 7: e39382.

Lampinen, R., Belaya, I., Boccuni, I., Malm, T., Kanninen, K. M. Mitochondrial Function in Alzheimer's Disease: Focus on Astrocytes. In Astrocyte - Physiology and Pathology. Gentile, MT., editor. Intech open 2018; p. 139-162.

Lane, C. A., Hardy, J., Schott, J. M. Alzheimer's disease. Eur J Neurol. 2018; 25: 59-70.

Liddelow S.A., Guttenplan K.A., Clarke L.E., Bennett F.C., Bohlen C.J., Schirmer L., Bennett M.L., Münch A.E., Chung W.S., Peterson T.C., Wilton D.K., Frouin A., Napier B.A., Panicker N., Kumar M., Buckwalter M.S., Rowitch D.H., Dawson V.L., Dawson T.M., Stevens B., Barres B.A. Neurotoxic reactive astrocytes are induced by activated microglia. Nature 2017; 541: 481-87.

Loppi S., Korhonen P., Bouvy-Liivrand M., Caligola S., Turunen T.A., Turunen M.P., Hernandez de Sande A., Kołosowska N., Scoyni F., Rosell A., García-Berrocoso T., Lemarchant S., Dhungana H., Montaner J., Koistinaho J., Kanninen K.M., Kaikkonen M.U., Giugno R., Heinäniemi M., Malm T. Peripheral inflammation preceeding ischemia impairs neuronal survival through mechanisms involving miR-127 in aged animals. Aging Cell. 2021;20:e13287. 
668

669

670

671

672

673

674

675

676

677

678

679

680

681

682

683

684

685

686

687

688

689

690

691

Luo S, Valencia C.A, Zhang J, Lee N.C, Slone J, Gui B, Wang X, Li Z, Dell S, Brown J, Chen

S.M, Chien Y.H, Hwu W.L, Fan P.C, Wong L.J, Atwal P.S, Huang T. Biparental inheritance of mitochondrial DNA in humans. Proc Natl Acad Sci U S A 2018, 115, 13039-44.

Morales, I., Sanchez, A., Puertas-Avendaño, R., Rodriguez-Sabate, C., Perez-Barreto, A., Rodriguez, M. Neuroglial transmitophagy and Parkinson's disease. Glia 2020; 68, 2277-99.

Nachlas, M. M., Tsou, K. C., de Souza, E., Cheng, C. S., Seligman, A. M. Cytochemical demonstration of succinic dehydrogenase by the use of a new p-nitrophenyl substituted ditetrazole. J Histochem Cytochem. 1957; 5: 420-36.

Oakley, H., Cole, S. L., Logan, S., Maus, E., Shao, P., Craft, J., Guillozet-Bongaarts A., Ohno M., Disterhoft J., Van Eldik L., Berry, R., Vassar, R. Intraneuronal $\beta$-amyloid aggregates, neurodegeneration, and neuron loss in transgenic mice with five familial Alzheimer's disease mutations: Potential factors in amyloid plaque formation. J Neurosci. 2006; 26: 10129-40.

Oksanen, M., Petersen, A. J., Naumenko, N., Puttonen, K., Lehtonen, Š., Gubert Olivé, M., Shakirzyanova A., Leskelä S., Sarajärvi T., Viitanen M., Rinne J.O., Hiltunen M., Haapasalo A., Giniatullin R., Tavi P., Zhang S., Kanninen K.M., Hämäläinen R.H., Koistinaho, J. PSEN1 Mutant iPSC-Derived Model Reveals Severe Astrocyte Pathology in Alzheimer's Disease. Stem Cell Reports 2017; 9: 1885-97.

Pickett, E. K., Rose, J., McCrory, C., McKenzie, C. A., King, D., Smith, C., Gillingwater T.H., Henstridge C.M., Spires-Jones, T. L. Region-specific depletion of synaptic mitochondria in the brains of patients with Alzheimer's disease. Acta Neuropathol. 2018; 136: 747-57.

Pickford, F., Masliah, E., Britschgi, M., Lucin, K., Narasimhan, R., Jaeger, P. A., Small S., Spencer B., Rockenstein E., Levine B., Wyss-Coray, T. The autophagy-related protein beclin 1 shows reduced expression in early Alzheimer disease and regulates amyloid $\beta$ accumulation in mice. J Clin Invest. 2008; 118: 2190-99. 
692

693

694

695

696

697

698

699

700

701

702

703

704

705

706

707

708

709

710

711

712

713
Reddy, P. H., Oliver, D. M. Amyloid Beta and Phosphorylated Tau-Induced Defective Autophagy and Mitophagy in Alzheimer's Disease. Cells 2019; 8: 488.

Rustom, A., Saffrich, R., Markovic, I., Walther, P., Gerdes, H. H. Nanotubular Highways for Intercellular Organelle Transport. Science 2004; 303: 1007-10.

Sarkar, P., Zaja, I., Bienengraeber, M., Rarick, K. R., Terashvili, M., Canfield, S., Falck J.R., Harder, D. R. Epoxyeicosatrienoic acids pretreatment improves amyloid-induced mitochondrial dysfunction in cultured rat hippocampal astrocytes. Am J Physiol Heart Circ Physiol 2014; 306: 475-84.

Soundara Rajan, T., Gugliandolo, A., Bramanti, P., Mazzon, E. Tunneling Nanotubes-Mediated Protection of Mesenchymal Stem Cells: An Update from Preclinical Studies. Int J Mol Sci. 2020; $21: 3481$.

Spees, J. L., Olson, S. D., Whitney, M. J., Prockop, D. J. Mitochondrial transfer between cells can rescue aerobic respiration. Proc Natl Acad Sci U S A 2006; 103: 1283-88.

Strappazzon, F., Nazio, F., Corrado, M., Cianfanelli, V., Romagnoli, A., Fimia, G. M., Campello S., Nardacci R., Piacentini M., Campanella M., Cecconi, F. AMBRA1 is able to induce mitophagy via LC3 binding, regardless of PARKIN and p62/SQSTM1. Cell Death Differ. $2015 ; 22: 419-32$.

Sun, X., Wang, Y., Zhang, J., Tu, J., Wang, X. J., Su, X. D., Wang L., Zhang, Y. Tunnelingnanotube direction determination in neurons and astrocytes. Cell Death Dis.2012; 3: e438e38.

Tiihonen, J., Koskuvi, M., Lähteenvuo, M., Virtanen, P. L. J., Ojansuu, I., Vaurio, O., Gao Y., Hyötyläinen I., Puttonen K.A., Repo-Tiihonen E., Paunio T., Rautiainen M.R., Tyni S., 
van Gijsel-Bonnello, M., Baranger, K., Benech, P., Rivera, S., Khrestchatisky, M., de Reggi, M., mouse model of Alzheimer's disease: Alleviation by pantethine. PLoS ONE 2017; 12: e0175369. PLOS ONE 2016; 11: e0150441.

Wang, W., Zhao, F., Ma, X., Perry, G., \& Zhu, X. Mitochondria dysfunction in the pathogenesis of Alzheimer's disease: recent advances. Mol Neurodegener. 2020; 15: 30. activating the JAK/STAT signaling pathway in human articular chondrocytes. Arthritis Rheum. 2009; 60: 792-800.

Yan, X., Shi, Z. F., Xu, L. X., Li, J. X., Wu, M., Wang, X. X., Jia M., Dong L.P. Yang S.H., Yuan, F. Glutamate Impairs Mitochondria Aerobic Respiration Capacity and Enhances Glycolysis in Cultured Rat Astrocytes. Biomed Environ Sci. 2017; 30, 44-51.

Yao, Y., Huang, J. Z., Chen, Y., Hu, H. J., Tang, X., Li, X. Effects and mechanism of amyloid $\beta 1$ 42 on mitochondria in astrocytes. Mol Med Rep. 2018; 17: 6997-74. and Alzheimer's disease patient brains. Hum Mol Genet. 2015; 24: 2938-51. 